\title{
Submitting Pathologist
}

National Cancer Institute

\section{Source}

National Cancer Institute. Submitting Pathologist. NCI Thesaurus. Code C51882.

A pathologist that submits a proposal or letter of intent to participate in a study or who submits results or diagnoses for use in a study. 